\title{
Management of External Inflammatory Cervical Root Resorption Using CBCT And MTA: A Case Report.
}

\author{
Satyajit Mohapatra. MDS ${ }^{1}$ \\ Govind Shashirekha.MDS ${ }^{2}$ Amit jena.MDS ${ }^{3}$ A Mahaprasad.BDS ${ }^{4}$ \\ ${ }^{1}$ Private Practice Conservative Dentistry \& Endodontics \\ ${ }^{2}$ Professor Conservative Dentistry \& Endodontics, Institute Of Dental Sciences, Siksha 'O' Anusandhan \\ University, Bhubaneswar-751003, Odisha,India \\ ${ }^{3}$ Professor \& HOD Conservative Dentistry \& Endodontics, Institute Of Dental Science, Siksha 'O' Anusandhan \\ University, Bhubaneswar-751003, Odisha,India \\ ${ }^{4}$ Post Graduate Trainee Conservative Dentistry \& Endodontics, Institute Of Dental Sciences, \\ Siksha 'O'Anusandhan University, Bhubaneshwar-751003, Odisha, India
}

\begin{abstract}
Invasive cervical resorption (ICR) is an uncommon form of external root resorption that is often aggressive in nature and often leads to loss of the tooth. This case report describes the successful management of external inflammatory ICR which occurred following trauma to the permanent dentition using cone-beam computed tomography (CBCT) and mineral trioxide aggregate (MTA).
\end{abstract}

Keywords: Invasive cervical resorption (ICR), Cone Beam Computed Tomography (CBCT), Mineral Trioxide Aggregate (MTA).

\section{Introduction}

Root resorption is an essential phenomenon that plays a crucial role in the physiologic and dynamic process of tooth eruption. Root resorption in the permanent dentition is an unwanted and pathological process that may occur due to various reasons. Biological mechanisms protecting against tooth resorption include the cementum and predentin layer present along the root surfaces that prevent the attachment of the osteoclasts. The major receptors for the binding of osteoclasts are the RGD - peptides bound to the calcium salt crystals on the mineralized surfaces. Thus, cementum along with a layer of cementoblasts along the outer surface prevents the attachment of osteoclasts (1). Hence, for root resorption, the two prerequisite factors required include loss or damage to the protective layer along the root surfaces (Cementum or predentin layer) and inflammation must occur to the unprotected root surfaces (1).

Damage to the protective layer can occur as a result of trauma or excessive orthodontic forces. Root resorption occurs in two phases. a) Destructive Phase: Where active resorption occurs between the multinucleated osteoclastic cells and the dried out cells and, b) Healing Phase: if the cementoblasts are able to recover, healing occurs and has a good prognosis. However, if the bone forming cells (osteoblasts) migrate into the resorptive areas and form bone, indicates a poor prognosis.

Classifying root resorption was first attempted by Andereasen(2) as Internal resorption (including inflammatory \& replacement) and External ( including surface, inflammatory \& replacement). Other forms of resorption that have been identified include transient apical internal resorption and hyperplastic resorptions. The hyperplastic resorptions can be invasive coronal, invasive cervical or invasive radicular types (3). Alternative classifications as proposed by Lindskog (4) include: trauma induced tooth resorption, infection induced tooth resorption and hyperplastic invasive tooth resorptions.

\section{Case Report}

A 29-year-old male healthy patient reported to the Department with the chief complaint of mobility in the upper and lower front tooth region of the jaw for the past one year. He revealed a previous history of trauma following a road traffic accident for which he had undergone extraction, intermaxillary fixation, and root canal therapy. Intraoral examination revealed a missing 21 with root canal treated 11,12 and 22. Miller's grade I mobility in 11, 12, 31, 32 and grade II mobile 42. Radiographic examination of 11,12 and 22 revealed radiopaque filling in the root canals, suggestive of previous nonsurgical root canal therapy. Loss of interdental bone was seen in relation to 11 and 12 and radiolucent area apical to the cementoenamel junction in relation to 12 , suggestive of external root resorption (Figure 1). A cone beam computed tomography was performed to access the extent of the lesion as well as to detect intracanal perforation (Figure 2 and 3). 


\section{Management:}

Mucosa in relation to 11 and 12 was anesthetized using local infiltration with local anesthetic containing adrenaline (Lox 2\%, Neon Laboratories Ltd, Mumbai). A full thickness mucoperiosteal flap was raised in relation to 22 to expose the defect (Figure 4) and granulation tissue present was curetted. Root conditioning was done using a mixture of tetracycline and dexamethasone (Dexarid, containing dexamethasone phosphate 4mg, Ridley Life Sciences, Delhi) (Figure 5). The resorptive defect was then repaired with white MTA (Angelus, Brazil). A radiograph was then taken to confirm the MTA placement (Figure 6). The flap was repositioned and sutured using interrupted sutures (Ethicon 3-0 Mersilk, Johnson \& Johnson, Himachal Pradesh).

\section{Discussion}

Invasive cervical resorption presents a clinical challenge and the prognosis depends on the extent of the resorptive process. One of the major drawbacks of using intra-oral periapical radiographs is the fact that they reveal only limited diagnostic information (5) since three dimensional structures are obtained on a two dimensional image (6). CBCT produces a three dimensional image of the anatomical structures. Patel S et al (6) compared the diagnostic accuracy of intra-oral peri-apical radiographs and CBCT in the diagnosis and management of teeth with root resorption and concluded CBCT to be superior. Thus, in the present case a CBCT was done to assess the extent of the resorptive lesion and to rule out any intra-canal communication by the resorptive process

Traditional management strategies include surgical exposure of the resorptive defect, curettage of the granulation tissue and restoration of the defect using appropriate materials $(7,8)$. Chemical cauterization of the granulation tissue using an aqueous solution of $90 \%$ trichloroacetic acid has been recommended (3). However, in this case, $90 \%$ aqueous solution of trichloroacetic acid was not used because isolation of the surrounding tissues in the surgical area could not be maintained as a result of the localization of the defect. Pharmacological management of ICR involves the topical application of tetracycline and dexamethasone (1). Tetracycline has an antimicrobial effect along with anti-resorptive effects. Topical application of corticosteroid has also been proved to have an anti-inflammatory effect and inhibit osteoclast-mediated resorption. Thus, in this case, a combination of tetracycline and dexamethasone $(4 \mathrm{mg})$ was used. MTA was chosen as the material of choice for restoration of the defect caused by ICR as it has good biocompatibility, sealability, low cytotoxicity,ability to set in presence of moisture and induction of a favorable tissue response $(9,10)$.

\section{Conclusion}

Successful management of ICR requires a prompt diagnosis and treatment owing to the severity of the disease process. With the advent of advanced diagnostic methods such as CBCT and biocompatible materials such as MTA, treatment of ICR has become more predictable.

\section{References}

[1]. Trope M. Root Resorption due to Dental. Endod Top. 2002;1(1):79-100.

[2]. Andreasen JO. Luxation of permanent teeth due to trauma. A clinical and radiographic follow-up study of 189 injured teeth. Scand J Dent Res; 1970;78(3):273-86.

[3]. Heithersay GS. Management of tooth resorption. Aust Dent J. 2007;52(1):105-21

[4]. Lindskog S, Heithersay GS PA. Dental resorptions. Scandinavi. Munksgaard: Blackwell Publishing Ltd; 2006.

[5]. Cohenca N, Simon JH, Mathur A, Malfaz JM. Clinical indications for digital imaging in dento-alveolar trauma. Part 2: root resorption. Dent Traumatol; 2007 Apr;23(2):105-13.

[6]. Patel S, Dawood A, Whaites E, Pitt Ford T. New dimensions in endodontic imaging: part 1. Conventional and alternative radiographic systems. Int Endod J; 2009 Jun;42(6):447-62.

[7]. Hiremath H, Yakub SS. Invasive Cervical Resorption : A Case Report. J Endod. 2007;33(8):999-1003.

[8]. Vinothkumar TS, Tamilselvi R. Reverse Sandwich Restoration for the Management of Invasive Cervical Resorption : A Case Report. J Endod [Internet]. Elsevier Ltd; 2011;37(5):706-10. Available from: http://dx.doi.org/10.1016/j.joen.2011.02.005

[9]. Parirokh M, Torabinejad M. Mineral trioxide aggregate: a comprehensive literature review--Part I: chemical, physical, and antibacterial properties. J Endod [Internet]. Elsevier Ltd; 2010;36(1):16-27. Available from: http://www.ncbi.nlm.nih.gov/pubmed/20003930

[10]. Torabinejad M, Parirokh M. Mineral Trioxide Aggregate: A Comprehensive Literature Review-Part II: Leakage and Biocompatibility Investigations. J Endod. Elsevier Ltd; 2010;36(2):190-202.

[11]. Management of External Inflammatory Cervical Root Resorption Using CBCT and MTA: A Case Report. 


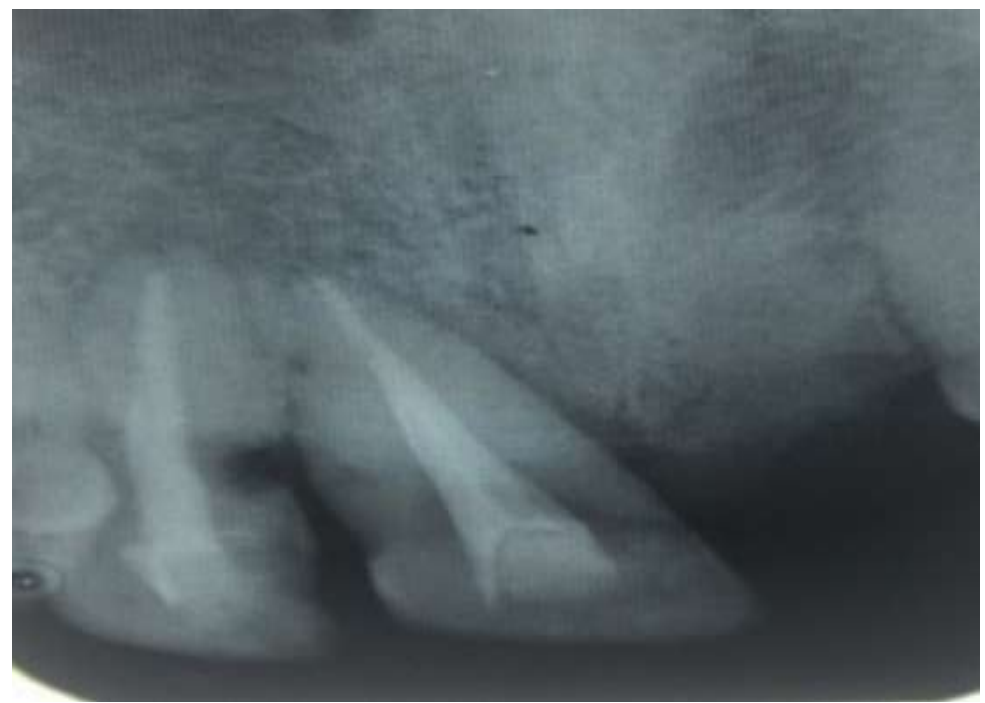

Figure 1: Preoperative Radiograph

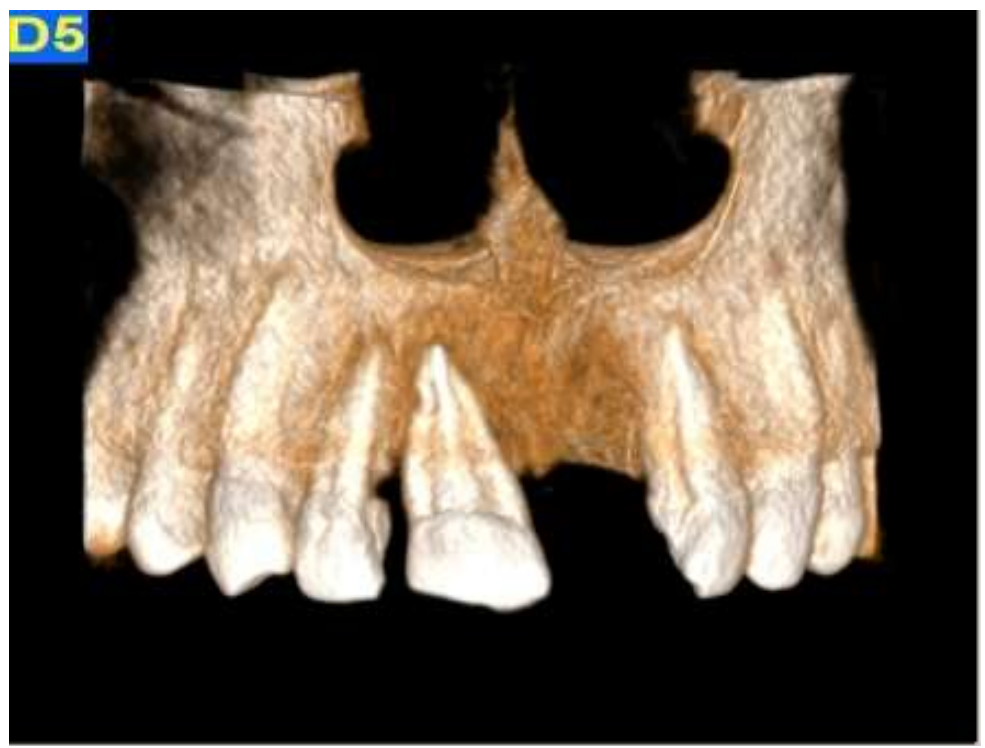

Figure 2

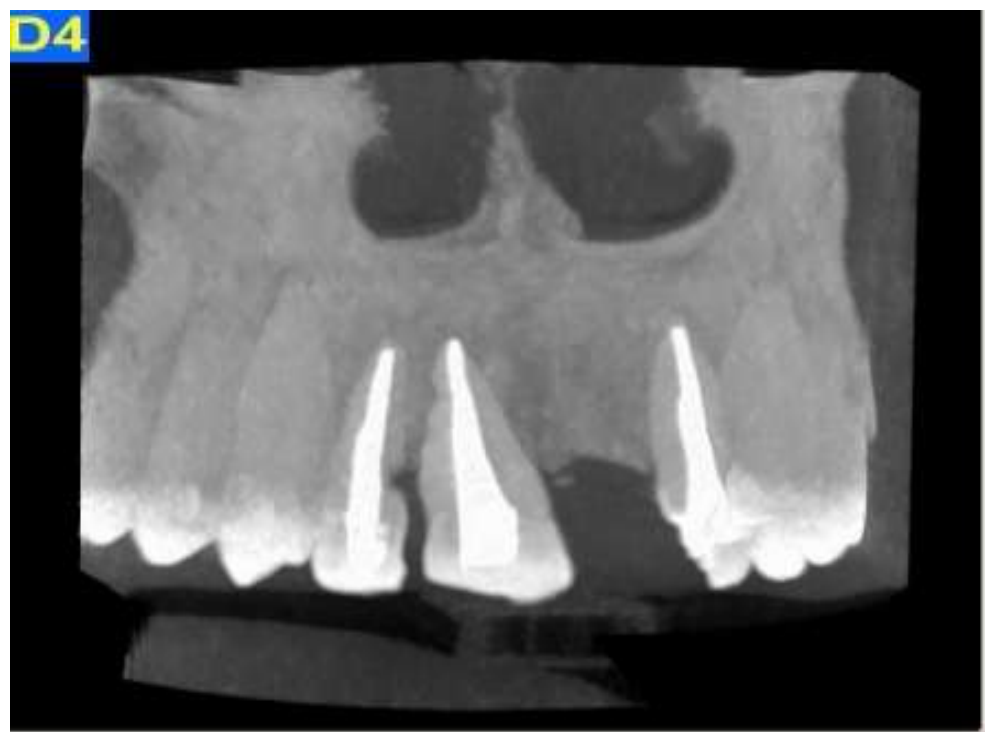

Figure 3 


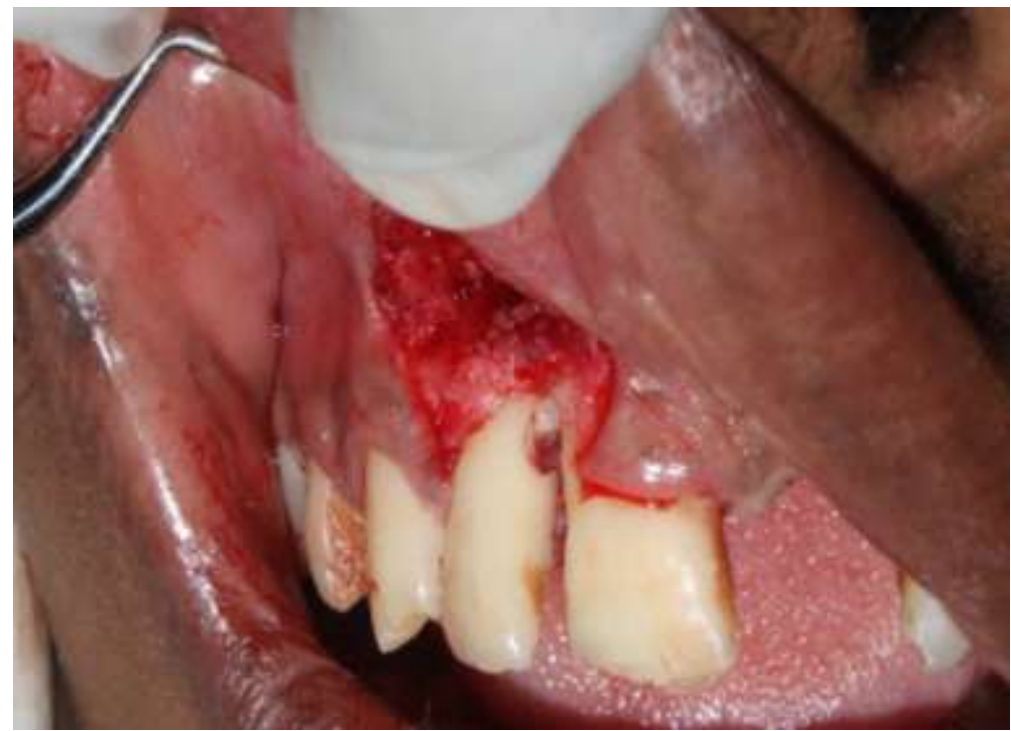

Figure 4: Surgical exposure of the resorptive defect

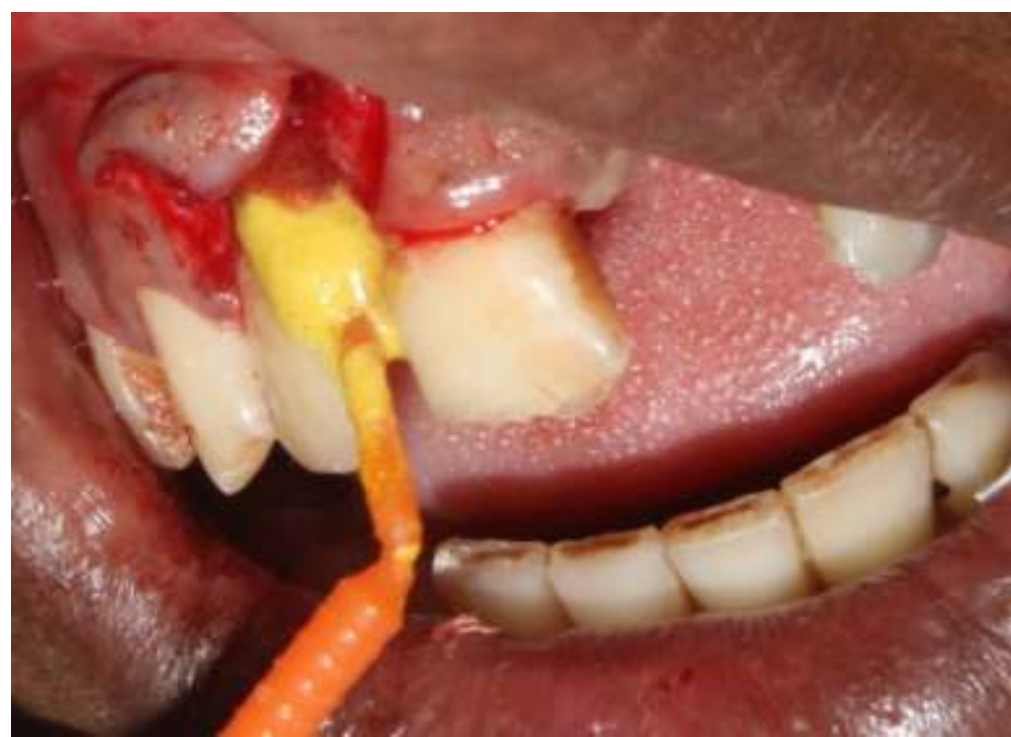

Figure 5: Root conditioning using tetracycline and dexamethasone

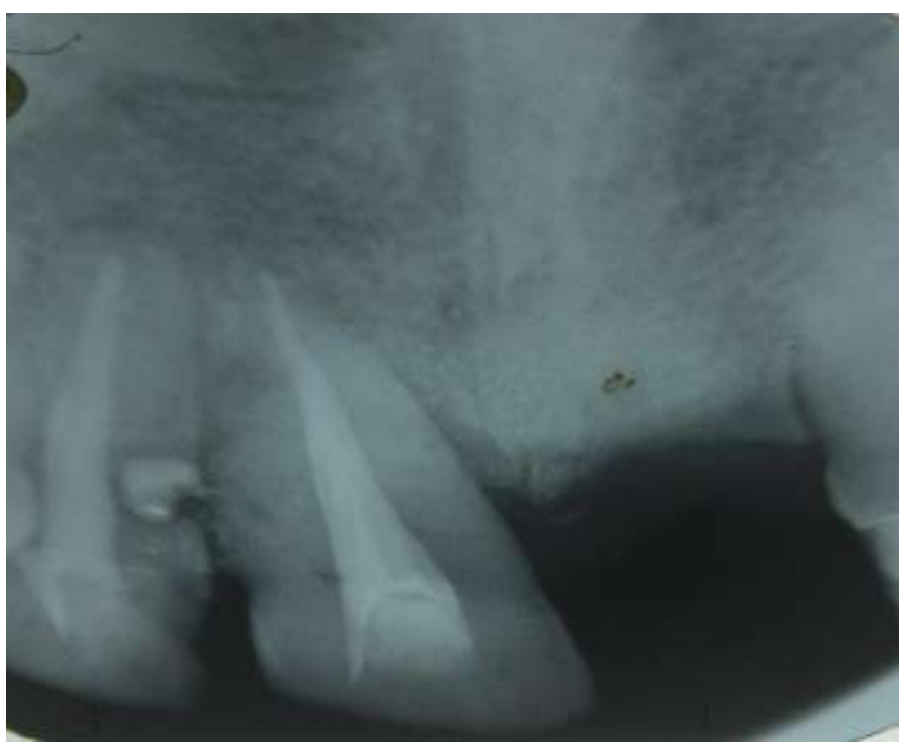

Figure 6: White MTA placed 